\title{
«Wir wollen die Wirklichkeit darstellen»
}

\author{
Ab 1. Januar 2012 wird in den Schweizer Spitälern mit diagnosebezogenen Fall- \\ pauschalen (DRG) abgerechnet. Der bevorstehende Systemwechsel wirft seine Schat- \\ ten voraus und führt zu engagierten Diskussionen über Aufgaben und Ziele der Medi- \\ zin. Verina Wild und Eliane Pfister gehören zum Team des Instituts für Biomedizinische \\ Ethik der Universität Zürich, das eine ethische Begleitforschung zur Einführung der \\ DRG in der Schweiz durchführt. Kürzlich haben sie ein Buch dazu veröffentlicht.
}

Wie sind Sie auf die Idee gekommen, das neue Vergütungssystem über DRG aus ethischer Perspektive zu untersuchen?

Verina Wild: Ich habe als junge Ärztin in Bremen und Berlin die Einführung der DRG hautnah miterlebt. In Berlin, wo der Spitalmarkt besonders umkämpft ist, benutzten die Spitalleitungen das DRG-System schnell dazu, die Schraube anzuziehen. Der Druck, Erlöse zu erwirtschaften, nahm zu, und wir Ärztinnen und Ärzte spürten, dass das Menschliche an unserer Arbeit in Gefahr geriet. Mir wurde bald klar, dass dieser Systemwechsel wichtige ethische Fragen beinhaltet. So beruht meine Motivation einerseits auf persönlichen Erfahrungen, andererseits aber auch auf Gesprächen mit Kolleginnen und Kollegen in Deutschland, die ich auch jetzt noch häufig führe. Als ich dann in die Schweiz kam, war ich überrascht und erfreut zu sehen, wie viele Leute bereits für das Thema DRG sensibilisiert sind und welch intensive Diskussionen dazu im Gang sind.

Eliane Pfister: Ich habe Philosophie, Neurobiologie und Volkswirtschaft studiert und mich haben die Schnittstellen zwischen Medizin, Ethik und Öko- nomie schon immer interessiert. Die Fallpauschalen haben Auswirkungen auf verschiedenen Ebenen des Gesundheitswesens. Nachdem ich seine Brisanz entdeckt hatte, liess mich das Thema nicht mehr los. Nun ist die Konzeption einer ethischen Begleitforschung zur Einführung der DRG in der Schweiz ein Teil meiner Dissertation.

Lassen sich die Erfahrungen aus Deutschland ohne weiteres auf die Schweiz übertragen?

Wild: Was das technische Konzept hinter den DRG betrifft, also mehr Wettbewerb, Kostentransparenz und Benchmarking, sind die beiden Länder ohne weiteres vergleichbar. Weniger eindeutig gilt das für die Auswirkungen: Es besteht Hoffnung, dass diese hierzulande weniger dramatisch ausfallen werden. Ich denke, der Druck, wie wir ihn in Deutschland gespürt haben, wird in den Schweizer Spitälern zumindest am Anfang weniger spürbar sein. Die Frage ist, ob der Moment kommt, an dem das System kippt. Dann müssen wir gewappnet sein für die Diskussion, ob wir weitermachen wollen oder nicht. Unter anderem dafür, dass wir diesen Moment nicht verpassen, ist unsere Forschung
Korrespondenz:

Dr. med. Verina Wild Institut für Biomedizinische Ethik

Ethik-Zentrum der Universität Zürich

Pestalozzistrasse 24

CH-8032 Zürich

Tel. 0446348371

wild@ethik.uzh.ch

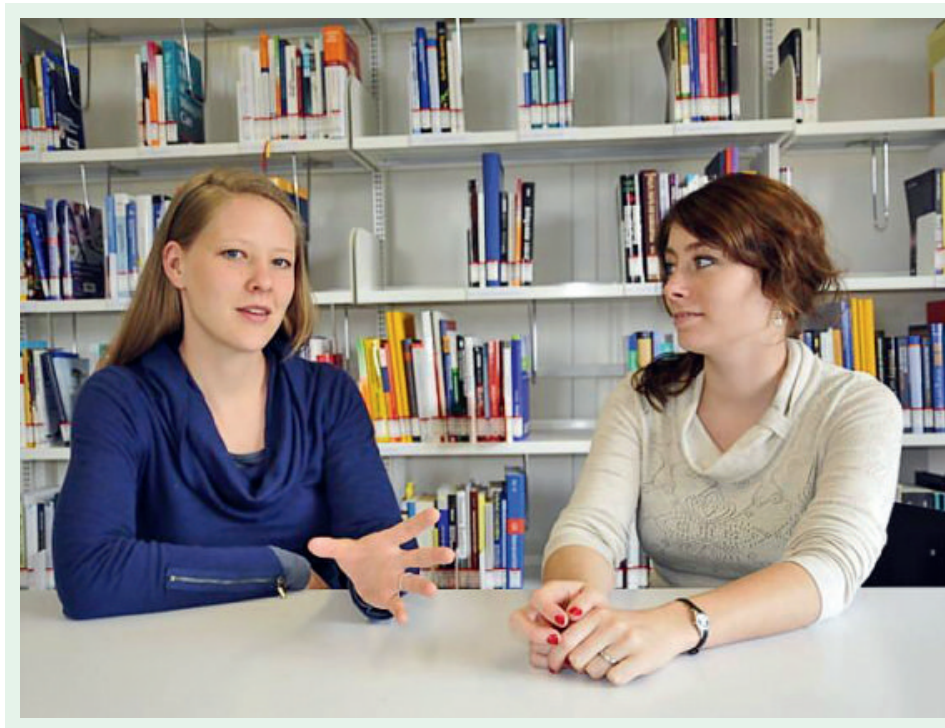

Im Gespräch mit der SÄZ:

Verina Wild (li.), Dr. med., geb. 1977, Studium der Humanmedizin an der Georg-August-Universität Göttingen. Dissertation über medizinethische Aspekte von Arzneimittelforschung an schwangeren Frauen. Arbeit als Ärztin in der Inneren Medizin in Berlin. Derzeit Oberassistentin am Institut für Biomedizinische Ethik der Universität Zürich.

Eliane Pfister (re.), lic. phil., Studium der Philosophie, Neurobiologie und Volkswirtschaft an den Universitäten Fribourg, Schweiz und Southampton, England. Sie ist seit 2007 wissenschaftliche Mitarbeiterin und Doktorandin am Institut für Biomedizinische Ethik der Universität Zürich und beschäftigt sich im Rahmen ihrer Dissertation mit dem Thema DRG. 
da, auch um Öffentlichkeit herzustellen, damit die Gesellschaft mitverfolgen kann, was geschieht.

Das System kippt? Was sollen wir uns darunter vorstellen?

Wild: Das ist der Moment, bei dem unerwünschte negative Folgen die angestrebten positiven Effekte dominieren. Wir können zum Beispiel die inneren Konflikte, denen das Gesundheitspersonal ausgesetzt ist, als Indikator nehmen. So führen wir in der ethischen Forschung qualitative Interviews oder speziell angepasste quantitative Studien durch, um herauszufinden, ab welchem Punkt es die Pflegenden, die Ärztinnen und Ärzte nicht mehr aushalten. Standardisierte Qualitätserhebungen der medizinischen Versorgung können noch über längere Zeit gute Werte ergeben, weil sich die Leute extrem anstrengen, um die Patienten gut $\mathrm{zu}$ betreuen. Aber niemand kann ernsthaft wollen, dass die Ärztinnen und Pflegenden ständig ihr Letztes geben müssen, um die Qualität auf einem akzeptablen Niveau zu halten. Diesen Punkt thematisieren wir auch in dem letzten Kapitel unseres Buches.

Handelt es sich beim Streit um die Einführung der Swiss$D R G$ vielleicht um eine Ersatzdiskussion für das allgemeine Unbehagen über die Ökonomisierung im Gesundheitswesen?

Pfister: Der Begriff der Ökonomisierung ist unter Gesundheitsfachleuten häufig negativ konnotiert: Wenn sie mit etwas nicht einverstanden sind, sprechen sie oft von Ökonomisierung. Ich nehme ein Unbehagen wahr, und es mag sein, dass zu viel davon den DRG zugeschrieben wird. Vielleicht müsste man wendigkeit von festetablierter Begleitforschung im Gesundheitswesen auslösen werden.

Auch Tagespauschalen und Einzelleistungsvergütungen setzen Anreize. In der laufenden Diskussion entsteht aber manchmal der Eindruck, ökonomische Anreize in der Medizin hätten in der Zeit vor DRG und Managed Care keine Rolle gespielt.

Pfister: Natürlich gab es früher auch schon Anreize. Jetzt aber werden neue Anreize gesetzt, die teils in die entgegengesetzte Richtung wirken. Diese Wirkungen muss man untersuchen.

Wild: Es kommt zu einer nie dagewesenen Transparenz. Für jeden einzelnen Arzt kann festgestellt werden, ob er wirtschaftlich arbeitet oder nicht. Damit entsteht ein persönlicher Druck, der vor allem die wirtschaftliche Arbeitsweise in den Vordergrund rückt. Wenn Spitalmanager wollen, können sie jederzeit genau sehen, wo die besten Erträge anfallen. Sie könnten also versuchen, die Aufnahme und Behandlung von Patientinnen so zu steuern, dass die Rechnung stimmt. Für mich als Ärztin fühlt sich das komisch an.

Komisch weshalb?

Wild: Weil es dann so wirkt, als wäre die wirtschaftliche Performance wichtiger als alles andere. Der Faktor, dass Menschen krank sind und es auch bleiben, bildet dabei die Grundlage für das Geschäft der Spitäler: Nur mit kranken Menschen können Gewinne erzielt werden.

Aber das ist doch nichts Neues.

Wild: Neu ist die Kundenlogik und die verbesserte Führung von Spitälern nach betriebswirtschaftlichen Kriterien. Ohne ein solches Anreizsystem, wie die

\section{«In die Erarbeitung des ethischen Rahmens und die Darstellbarkeit durch geeignete Indikatoren ist viel Vorbereitungszeit geflossen.»}

daher eher von einer «Projektionsfläche» sprechen. «Ersatzdiskussion» ist aber sicher das falsche Wort, denn die DRG verdienen trotz allem eine genaue Untersuchung.

Wild: Ich denke auch, dass DRG eine besondere Beachtung verdienen. Ich kenne kein vergleichbares Instrument, das die Spitallandschaft, die Arbeit der Menschen in Gesundheitsberufen, die medizinische Vor- und Nachsorge und die Ausbildung der Medizinerinnen und Mediziner derart stark beeinflusst. Insgesamt sollte es aber doch eine Selbstverständlichkeit sein, dass Innovationen im Gesundheitswesen wissenschaftlich begleitet werden. Das gilt für die DRG ebenso wie für andere Anreizsysteme der ManagedCare-Strukturen. Wir haben uns die DRG als Forschungsobjekt vorgenommen, hoffen aber, dass wir damit auch eine Meta-Diskussion über die Not-
DRG es sind, ist es schwierig, diese Logik konsequent einzuführen.

Pfister: Grundsätzlich finde ich es nicht einfach falsch, wenn Ärztinnen und Ärzte mitverantwortlich sind für die Einhaltung des Budgets. Niedergelassene Ärzte kennen das schon lange. Die Frage ist aber, auf wie vielen Schultern die Verantwortung verteilt ist und wie viel Druck erträglich ist, auch bezüglich der Auswirkungen auf die Patienten. Ich habe stark den Eindruck, dass entscheidend ist, wie ein Spital geführt ist und wie die DRG konkret umgesetzt werden, also ob zum Beispiel Spielraum bleibt für Lösungen, die der einzelnen Patientin möglichst gerecht werden.

Spielraum gibt es dann, wenn genügend Geld im System ist. Ist es nicht letztlich eine Frage der Ressourcen, ob Patienten und Personal Schaden nehmen oder nicht? 
Pfister: Ich bin nicht einverstanden mit der Aussage, so lange genug Geld da sei, spiele es keine Rolle, welches System wir haben. Auswirkungen innerhalb des Systems sind trotzdem vorhanden, und wir müssen den Einsatz und die Verschiebung von Ressourcen im Auge behalten. Vorläufig haben wir in der Schweiz wahrscheinlich aber noch Spielraum.

Wild: An dieser Stelle möchte ich einmal festhalten, dass es uns nicht darum geht, die DRG schlechtzumachen. Es gibt auch gute Effekte.

\section{Worum geht es Ihnen?}

Wild: Es geht uns um die wissenschaftliche, also ergebnisoffene Begleitung der Einführung der DRG. Wir möchten mit Hilfe von geeigneten Indikatoren die Arbeitswirklichkeit und die Versorgung im Gesundheitswesen darstellen, also für Transparenz in diesem Bereich sorgen. Diese Ergebnisse möchten wir mit einem ethischen Rahmen abgleichen, den wir erarbeiten. Da geht es auf der Makroebene um grundlegende Werte wie soziale Gerechtigkeit und Verteilungsgerechtigkeit. Auf der Mesoebene geht es um ethisches Handeln in Organisationen. Auf der Mikroebene geht es um individuelle Menschen in Gesundheitsberufen und ihre ethischen Massstäbe im Umgang mit Patienten wie etwa patientenzentrierte Fürsorge und Nichtschaden. In die Erarbeitung des

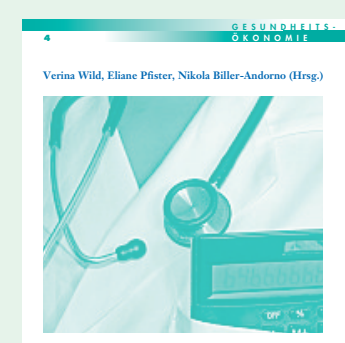

DRG und Ethik

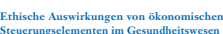

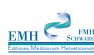

Verina Wild, Eliane Pfister, Nikola Biller-Andorno (Hrsg.) DRG und Ethik.

Ethische Auswirkungen von ökonomischen Steuerungselementen im Gesundheitswesen

Basel: EMH Schweizerischer Ärzteverlag; 2011

235 Seiten. 38 CHF.

ISBN 978-3-03754-052-7

\section{Neues Buch zu Ökonomie und Ethik}

\section{im Gesundheitswesen}

Das Fehlen einer systematischen Begleitforschung zur Einführung von DRG in der Schweiz trägt wesentlich zur kritischen Haltung vieler Berufsleute im Gesundheitswesen gegenüber dem neuen Spitalfinanzierungsmodell bei. Das Institut für Biomedizinische Ethik an der Universität Zürich unter der Leitung von Prof. Nikola Biller-Andorno will wenigstens einen Teil der Forschungslücken schliessen: Die Forscherinnen untersuchen die Auswirkungen der Spitalfinanzierung über Fallpauschalen aus ethischer Perspektive. Der nun erschienene Sammelband in der Reihe Gesundheitsökonomie des Ärzteverlags EMH bildet einen Zwischenschritt der Forschungsaktivitäten am Institut. Kürzlich ist hier auch ein interdisziplinäres und vom $\mathrm{Na}$ tionalfonds gefördertes DRG-Begleitforschungsprojekt angelaufen.

Das Buch schliesst und eröffnet gleichzeitig wieder zahlreiche Baustellen rund um die neuen Finanzierungsmodelle im Gesundheitswesen. Es ist allein deshalb lesenswert, weil es die omnipräsente ökonomische Forschung zu diesem Gebiet um ethische Fragestellungen erweitert. Die Forschenden und Gesundheitsfachleute, die im Buch zu Wort kommen, fragen nicht nur nach den Auswirkungen von DRG und Managed-Care-Modellen auf Fallkosten, Effizienz oder standardisierte Qualitätsziffern. Vielmehr stehen im Zentrum die Werthaltungen der Menschen, die im Gesundheitswesen arbeiten, ihre Überzeugungen, Menschenbilder und inneren Konflikte. Zugleich wirft das Buch zahlreiche neue Fragen auf, die noch zu bearbeiten wären, so zum Beispiel die Auswirkungen veränderter ökonomischer Anreize auf die Beziehungen zwischen Gesundheitspersonal und Patientinnen und Patienten. ethischen Rahmens und die Darstellbarkeit durch geeignete Indikatoren ist viel Vorbereitungszeit geflossen. Es wird eine andauernde Aufgabe für uns bleiben, den Rahmen und die Indikatoren zu diskutieren und zur Diskussion zu stellen. Implizit ist der Rahmen ja längst vorhanden: Dass die Medizin prinzipiell für alle gleichermassen zugänglich sein soll, darüber zum Beispiel sind sich die meisten einig. Wir arbeiten nun daran, diese Dinge explizit zu machen.

Die fehlende Begleitforschung dient den Befürwortern eines DRG-Moratoriums als wichtiges Argument. In den letzten Monaten sind jedoch mehrere Forschungsprojekte aufgegleist worden. Genügt das?

Pfister: Wir sind zwar weiter als vor ein, zwei Jahren, aber die Situation ist nach wie vor unbefriedigend. Was beispielsweise fehlt, aber wichtig wäre, ist eine ökonomische Begleitforschung, die zum Beispiel den Einfluss auf die Kostenentwicklung in den verschiedenen Sektoren der Gesundheitsversorgung untersucht. Leider haben wir in der Schweiz keine verlässlichen Gesundheitsdaten. Vielleicht bringen ja die DRG hier einen Fortschritt.

Sie haben bereits gesagt, dass die ethische Begleitforschung Ihres Instituts Transparenz schaffen will über die Auswirkungen der DRG. Wie dürfen wir uns das konkret vorstellen?

Wild: Die Erarbeitung des ethischen Rahmens, die Entwicklung von Kriterien für qualitative und quantitative Studien und die Erhebung der realen Bedingungen werden wir in unserem DRG-Begleitforschungsprojekt weiterverfolgen, das kürzlich angelaufen ist. In dem SNF-Projekt arbeiten wir mit Teams aus der Pflegewissenschaft, aus der Versorgungsforschung und aus dem Medizinrecht zusammen. Das Ganze ist ein dynamischer Prozess. Unser Buch ist ein Baustein auf diesem Weg: Im ersten Teil erhalten wir durch die Erfahrungsberichte einen Eindruck der tatsächlich erlebten Wirklichkeit einzelner Menschen. Anschliessend stellen wir verschiedene Studien vor, die sich wissenschaftlich mit dem Thema DRG beschäftigt haben, und im dritten Teil folgen theoretische Überlegungen dazu.

Pfister: Für die ganze Diskussion scheint mir wichtig, dass man sich bewusst ist, dass alle Beteiligten aus ihrer jeweiligen Perspektive heraus argumentieren: Ärztinnen, Pflegende, Spitalmanager, Ökonomen, Patienten und so weiter. Viele Sichtweisen hängen davon ab, welche Aufgaben man zu erfüllen hat, welche Erfahrungen man mit den DRG bereits gemacht hat und welche moralischen Überzeugungen man hat. Dagegen ist auch nichts einzuwenden. Es kommt so aber zu sehr unterschiedlichen Einschätzungen, die manchmal schwer auf einen gemeinsamen Nenner zu bringen sind. Die Ethik kann versuchen, die unterschiedlichen Perspektiven einzubinden und Anhaltspunkte $\mathrm{zu}$ finden, die eine gemeinsame, fruchtbare Diskussion ermöglichen. 